https://helda.helsinki.fi

\title{
Aging and non-native speech perception : A phonetic training study
}

\section{Tamminen, Henna}

2021-01-01

Tamminen , H , Kujala , T , Näätänen , R \& Peltola , M S 2021 , ' Aging and non-native speech perception : A phonetic training study ' , Neuroscience Letters , vol. 740 , 135430 . https://doi.org/10.1016/j.n

http://hdl.handle.net/10138/335567

https://doi.org/10.1016/j.neulet.2020.135430

cc_by_nc_nd

acceptedVersion

Downloaded from Helda, University of Helsinki institutional repository.

This is an electronic reprint of the original article.

This reprint may differ from the original in pagination and typographic detail.

Please cite the original version. 


\section{Journal Pre-proof}

Aging and non-native speech perception: A phonetic training study

Henna Tamminen (Conceptualization) (Methodology) (Formal analysis) (Investigation) (Data curation) (Writing - original draft) (Writing - review and editing) (Visualization) (Supervision) (Project

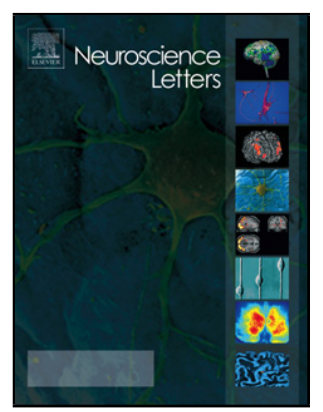
administration) (Funding acquisition), Teija Kujala (Data curation) (Writing - review and editing), Risto Näätänen (Data curation) (Writing - review and editing), Maija S. Peltola (Conceptualization) (Methodology) (Formal analysis) (Investigation) (Data curation) (Writing - review and editing) (Supervision) (Project administration)

PII:

S0304-3940(20)30700-X

DOI: https://doi.org/10.1016/j.neulet.2020.135430

Reference:

NSL 135430

To appear in:

Neuroscience Letters

Received Date:

14 March 2020

Revised Date: 18 September 2020

Accepted Date: 11 October 2020

Please cite this article as: Tamminen $\mathrm{H}$, Kujala T, Näätänen R, Peltola MS, Aging and non-native speech perception: A phonetic training study, Neuroscience Letters (2020), doi: https://doi.org/10.1016/j.neulet.2020.135430 
This is a PDF file of an article that has undergone enhancements after acceptance, such as the addition of a cover page and metadata, and formatting for readability, but it is not yet the definitive version of record. This version will undergo additional copyediting, typesetting and review before it is published in its final form, but we are providing this version to give early visibility of the article. Please note that, during the production process, errors may be discovered which could affect the content, and all legal disclaimers that apply to the journal pertain.

(C) 2020 Published by Elsevier. 


\title{
Aging and non-native speech perception: A phonetic training study
}

Henna Tamminen ${ }^{a}$, , ${ }^{*}$ henna.tamminen@utu.fi, Teija Kujalacteija.m.kujala@helsinki.fi, Risto Näätänen ${ }^{\mathrm{c}, \mathrm{d}, \mathrm{e}}$ risto.naatanen@ @elsinki.fi, Maija S. Peltola ${ }^{\mathrm{a}, \mathrm{b}}$ maija.peltola @utu.fi

aPhonetics and Learning, Age \& Bilingualism laboratory (LAB-lab), Department of Future Technologies, University of Turku, Turku, Finland

${ }^{\mathrm{b}}$ Centre for Cognitive Neuroscience, University of Turku, Turku, Finland

${ }^{c}$ Cognitive Brain Research Unit, Department of Psychology and Logopedics, Faculty of Medicine, University of Helsinki, Helsinki, Finland

${ }^{\mathrm{d}}$ Center of Functionally Integrative Neuroscience, Aarhus University, Aarhus, Denmark

${ }^{\text {e} I n s t i t u t e ~ o f ~ P s y c h o l o g y, ~ U n i v e r s i t y ~ o f ~ T a r t u, ~ T a r t u, ~ E s t o n i a ~}$

*Corresponding author: Henna Tamminen Phonetics and Learning, Age \& Bilingualism laboratory (LAB-lab), Department of Future Technologies Koskenniemenkatu 4 FIN-20500 Turku FINLAND.

\section{HIGHLIGHTS}

- seniors show training effects in the most attention demanding perceptual tasks

- no training effects in discrimination or neural perception

- experience-based linguistic knowledge benefits the elderly

\begin{abstract}
Cognitive decline is evident in the elderly and it affects speech perception and foreign language learning. A listen-and-repeat training with a challenging speech sound contrast was earlier found to be effective in young monolingual adults and even in advanced L2 university students at the attentive and pre-attentive levels. This study investigates foreign language speech perception in the elderly with the same protocol used with the young adults. Training effects were measured with attentive behavioural measures $(\mathrm{N}=9)$ and with electroencephalography measuring the pre-attentive mismatch negativity $(\mathrm{MMN})$ response $(\mathrm{N}=10)$. Training was
\end{abstract}


effective in identification, but not in discrimination and there were no changes in the MMN. The most attention demanding perceptual functions which benefit from experience-based linguistic knowledge were facilitated through training, whereas pre-attentive processing was unaffected. The elderly would probably benefit from different training types compared to younger adults.

Keywords: Training; Speech perception; Phonological processing; Aging; Mismatch negativity (MMN)

\section{Introduction}

Speech perception and foreign language learning are demanding cognitive processes which are affected by aging. They involve both conscious and subconscious processes that may be differently affected by age. Different training methods have been proven effective in foreign language speech perception and production in children and in adults. Positive training effects in children have been shown, for example, in production with listen-and-repeat training [1] and in behavioural perception with high-variability perceptual training and discrimination training $[2,3]$. Pre-attentive perception also improves in children as a result of listen-and-repeat training [4]. Adults benefit from perception and production training as well. For example, listen-andrepeat training on adults has shown that visual transcription cues, rather than orthographic cues, guide towards non-native target productions when presented with synchronous acoustic models [5] and specific pronunciation instructions were shown to be effective already after one training session [6]. Listen-and-repeat training has also proven to be effective for foreign language perception learning at both the pre-attentive and behavioural levels [7]. Furthermore, the same training was able to strengthen memory traces in advanced students [8].

Cognitive decline is evident in the elderly brain: brain structures change and shrink, white matter integrity decreases and dopamine, which is vital for learning [e.g., 9], depletes. Age- 
related compensatory scaffolding increases functional brain activity, compensating for declines in various processes, but these scaffolded networks are probably less efficient than the original networks. [10] Compensatory processing in elderly compared to young adults has been shown, for example, during spoken word processing in noise, shown also by positive correlation with behavioural performance, [11] and rhyme judgement tasks [12]. Spoken syntactic complexity is, however, not affected by aging [13], whereas semantic processing is [14]. Furthermore, age may [15] or may not [16] influence performance in verbal fluency when comparing different age groups among seniors.

In older adults, precision in perceiving temporal features is also reduced, compared to younger adults [17,18] and children [19]. These studies used plosive-vowel syllables varying in voice onset time (VOT) to measure both behavioural and psychophysiological discrimination. Strouse and colleagues [17] also tested identification which was not as systematic in older versus younger adults.

As it is evident that some cognitive functions decline and some native language linguistic processes change with age, foreign language speech perception learning and responsiveness to training are likely also influenced. Native language speech perception training seems to be effective for elderly adults with mild to moderate hearing loss, as word identification in noise and speech discrimination from background noise is better after training, compared to agematched-controls [20], but foreign language learning and training studies regarding phonological processes in speech perception and production on the elderly are scarce. However, it has been shown that elderly people who study foreign languages benefit from foreign language speech production training, while otherwise active seniors with interests other than languages, do not benefit from the training [21]. 
The sound contrast (/f/ - /v/) in this study represents two phonemes in English, whereas only /f/ is part of the Finnish phoneme repertoire. Finnish does not differentiate fricatives by voicing, and hence, the stimulus pair /fi:1/ - /vi:1/ exhibits a difficult perceptual contrast for Finns. The English /f/ - /v/ pair assimilates unequally into Finnish /f/ (/f/ better than /v/), causing difficulties according to the Perceptual Assimilation Model (PAM) [22]. According to the Speech Learning Model (SLM) [23], the English /v/ can also be described as similar to Finnish /f/, not identical or new, which again causes problems. As the English /v/ assimilates into Finnish /f/, it is difficult to perceive as distinct from /f/.

Our main aim was to investigate whether elderly adults, who are generally known to have reduced behavioural and neural plasticity due to aging, are still able to learn a non-native contrast. We exposed them to the non-native /v/ - /f/ contrast via a listen-and-repeat protocol. The contrast presents a feature that is phonologically relevant in the foreign target language, but not in the native language. During training, the participants repeated the target words after listening to them carefully. The effects of the training were measured both at the behavioural level with identification (ID), goodness rating (GR) and oddball discrimination tasks, and at the neural level with psychophysiological measures recording mismatch negativity (MMN) responses. The MMN is an automatically elicited neural change detection response which increases as a function of learning to detect stimulus differences [e.g., 24]. The exact same protocol has been used previously $[7,8]$ to measure training effects on young monolingual adults and on target language students, respectively. Training was effective on all measures but GR in the monolingual adults, and all but category boundary location and GR in the target language students. Our hypothesis is, given for example the results in the previously mentioned VOT studies $[17,18]$, that training may not lead to improvement in the behavioural tasks like it did in young adults [7]. Further, given the neural and cognitive changes related to aging, the 
pre-attentive perceptual performance of the elderly may not show as fast and drastic changes as in young monolingual adults [7] and advanced language learners [8].

\section{Methods}

\subsection{Participants}

Healthy, normally hearing, right-handed Finnish speakers participated in this study. All participants had been retired and off the working life for at least one year when tested. Originally, there were eleven participants, two of whom were excluded from the behavioural analyses and one from the electrophysiological analyses. Hence, there were data from 9 (4 females) 61 to 69-year-old (mean age 64.3) participants in the behavioural studies and 10 (5 females) 61 to 71-year-old (mean age 65) participants in the electroencephalography (EEG) experiments. Two participants did not manage the cognitive task in the behavioural tests as at least in one session they failed to press the appropriate buttons. The exclusion in the EEG data was due to a low amount of accepted deviant trials. The participants reported having no diagnosed neurological illnesses or medication affecting the central nervous system. Participants' hearing was tested with an audiometer with perceptually relevant frequencies of $250 \mathrm{~Hz}, 500 \mathrm{~Hz}, 1000 \mathrm{~Hz}, 2000 \mathrm{~Hz}$, and $4000 \mathrm{~Hz}$; all participants had normal hearing within this range. Handedness was tested with the Edinburgh Handedness Inventory [25]. All participants gave their informed consent prior to attending the experiments. The study was approved by the Ethics Committee of the University of Turku, Finland and was carried out in accordance with The Code of Ethics of the World Medical Association (Declaration of Helsinki). 
The participants had completed the Finnish primary education system in the 1950s or 1960s. On average, they had studied English for 3.3 years (range 0-7 years) during their primary education, and only one had studied English since. This participant reported having studied English for three years after primary education but not mastering speaking, understanding, reading, or writing at all. Three participants had never studied English and two of them had never studied any languages at all. For detailed information on the self-reports on English language skills, daily exposure to English, and years of English studies during primary education, see Table 1. Even though some participants had studied English decades before the experiment, they had extremely low pronunciation skills and knowledge. All participants had recreational activities, such as exercising, needlework, reading, and culture. The former occupations of the participants were print worker, secretary, teacher, managing agent, special education teacher (2), head of technical services, office employee, IT professional, and district heating specialist. In other words, they represented a normal population.

Insert Table 1 about here.

\subsection{Stimuli}

The stimuli (same as described in detail in [7]) for the behavioural ID and GR experiments consisted of 15 variants of the words /fi:l/ 'feel' and /vi:1/ 'veal' (synthesised using HLsyn software 1.0 Sensimetrics Inc.). Only the VOT of the labiodental fricative was varied. Hence, there was no voicing in the fricative at one end of the continuum, but voicing was present during the entire frication at the other end. Voicing was added in $14 \mathrm{~ms}$ steps from the vowel towards the beginning of the fricative. The overall duration of the stimuli was $499 \mathrm{~ms}$. Based on a pilot identification experiment with native English speakers, two stimuli representing 
different categories were selected from the continuum. These two stimuli were used in the discrimination, reaction time (RT), and EEG experiments as well as in training. The VOTs of the two stimuli were $113 \mathrm{~ms}$ and $71 \mathrm{~ms}$ for /fi:1/ and /vi:1/, respectively.

\subsection{Procedure and analysis}

The experiments and the research procedure are described in detail in [7]. In the forced choice, self-paced ID and GR task the participants identified the stimuli as either /fi:1/ or /vi:1/ and rated the goodness of it on a scale where 1 was poor and 7 was excellent. The participants heard the 15 stimuli repeated eight times in random order. To obtain the category boundary locations and the boundary steepness values, the ID data was subjected to a logit transformation analysis (statistical software SPSS). The boundary location is the point where the distribution of answers is $50 \%$. The steepness value indicates the tilting of the ID response curve, i.e., the consistency of the answers. The discrimination and RT experiment used an oddball paradigm where /fi:1/ was the standard and /vi:1/ the deviant stimulus, deviant probability being 0.13 (130 standards, 20 deviants per each block). Inter-stimulus interval was 1000 ms. RTs were measured from the onset of the stimulus and responses within \pm 3 standard deviations were included in the analysis. Discrimination sensitivity $\left(d^{\prime}\right)$ was calculated by using the hits, misses, false alarms, and correct rejections. Both experiments started with a short familiarisation block which data were not included in the analysis.

An oddball paradigm was also used in the EEG experiment. The stimuli were the same as in the discrimination test with the same deviant probability (783 standards, 120 deviants per each block). The participants were instructed to ignore the stimuli and to concentrate on watching a silenced and non-subtitled movie. The order of the discrimination and the EEG tests was counterbalanced across the participants. The EEG (Synamps amplifier; sampling rate $250 \mathrm{~Hz}$, 
bandwidth $0.5-70 \mathrm{~Hz}$ ) was nose-referenced and it was registered with $21 \mathrm{Ag} / \mathrm{AgCl}$ electrodes (Electro-Cap International, Inc.). Impedance was kept under $5 \mathrm{k} \Omega$ and electrodes attached below and near the outer canthus of the right eye monitored eye movements. The event-related potential (ERP) epochs (550 ms window including a $50 \mathrm{~ms}$ pre-stimulus period) were filtered off-line with a $1-30 \mathrm{~Hz}$ bandpass filter and artefact criterion was set at $\pm 100 \mu \mathrm{V}$. The baseline correction period was set from $50 \mathrm{~ms}$ before stimulus onset to $71 \mathrm{~ms}$ after stimulus onset, where the difference between the standard and the deviant stimuli started. Separately averaged waveforms for the standard and the deviant stimuli were computed for each participant. Difference waveforms were created by subtracting the standard response from the deviant response. The standards occurring right after the deviant were excluded from the analyses. A 300-360 ms time window (difference between the stimuli started at $71 \mathrm{~ms}$ ) and three frontal electrodes (Fz, F3, and F4) were selected for the mean amplitude analysis since the MMN is maximal over these areas [24].

During listen-and-repeat training the participants were instructed to listen to the stimuli and then repeat the words as accurately as possible. They were told to especially pay attention to the first consonant sound. The test was self-paced, and there were 30 /fi:1/ and 30 /vi:1/ stimuli played in turns, resulting in 60 words in every training session. No feedback was given in any of the four sessions of training and testing. In every experiment the stimuli were delivered binaurally via headphones (Sennheiser HD25) using Presentation software (Neurobehavioral Systems, Inc.).

The category boundary location and steepness were analysed separately with a Repeated Measures Analysis of Variance (ANOVA) (sphericity assumed). The GR data were statistically analysed for four stimuli (the trained stimuli 7 and 10 and two stimuli, 8 and 9, between them, i.e., the category boundary area) with a $2 \times 4$ ANOVA with Session (pre and post training) and Stimulus (7, 8, 9, and 10) as within-subjects factors. The RT and $d^{\prime}$ data were subjected 
separately to an ANOVA (sphericity assumed) comparing the pre and post training sessions. We examined the training effects on MMN with a $2 \times 3$ ANOVA (sphericity assumed) with Session (pre and post training) and Electrode (Fz, F3, F4) as within-subjects factors. The analyses were performed with IBM SPSS Statistics 23. Bonferroni adjustments were performed to correct for multiple comparisons.

\section{Results}

There were statistically significant findings between the pre and post training sessions. The category boundary was significantly $\left(F(1,8)=5.808, p=0.043 ; \eta_{p}^{2}=0.421\right)$ different when comparing the pre and post training sessions (Fig. 1.). The baseline boundary was at stimulus 10 (range 8.1-11.6, std. dev. 1.04), but after four sessions of training it was at 8.28 (range $4.7-$ 10.0, std. dev. 1.60). The consistency of the boundary did not change. The descriptive statistics of the boundary location and consistency are shown in Table 2. In the GR data, a significant interaction between Stimulus and Session $\left(F(3,24)=4.980, p=0.008 ; \eta_{p}{ }^{2}=0.384\right)$ was found. Paired samples t-tests showed no significant differences between sessions or within the pre training session. However, there was a significant difference between stimuli 9 and $10(\mathrm{t}(8)=-$ 3.214, $\mathrm{p}=0.012$ ) after training suggesting that the new boundary was rated differently from the trained stimulus. As the descriptive statistics show, the boundary stimulus was rated poorer than the trained stimulus of the /vi:1/ category. See Table 2 for the descriptive statistics of the GR data.

Insert Figure 1 about here. 
Insert Table 2 about here.

There were no significant findings in either the RTs or the $d$ 's, indicating that the training had no effects on either of them. The descriptive statistics are shown in Table 2. There were no significant findings in the MMN either, suggesting that the training was ineffective at the preattentive level of perception. The results are shown in Fig. 2 and Table 2.

-

Insert

Figure

2

about

here.

\section{Discussion}

The effects of a listen-and-repeat training on foreign language speech perception in elderly people at attentive and pre-attentive level were of interest in this study. The only significant effects were in the ID and GR. The training did not affect the behavioural or pre-attentive discrimination.

The present training paradigm has shown both attentive and pre-attentive effects on monolingual young adults [7] and advanced L2 university students [8]. Training of the two stimuli, and exposure to them and the whole continuum during the three days of testing, alters identification similarly in both the monolingual elderly participants of the present study and monolingual young adults [7]. In both studies, the category boundary shifted after training. The baseline boundary was near or at one of the trained stimuli, but after training it was between the trained stimuli. Another significant training-related change in the elderly appeared in the GR between stimuli 9 and 10 after four training sessions in the current study. Stimulus 10 was the baseline boundary, whereas stimulus 9 was the new boundary area in the post training measurement and was then rated poorer than the trained stimulus. This change in the GR, and 
category hierarchy, is interesting, since category boundary is typically rated as poor and the prototypical category representatives as good exemplars. In addition, the former boundary stimulus was now rated better than the new boundary. This shows that the participants not only changed the boundary location, but that they grew sensitive to hierarchical goodness estimates. In contrast, in our previous studies, the young adults showed no GR differences at all [7]. Proficient language students, however, showed differences between the different stimuli, not as a training effect [8].

Discrimination sensitivity and reaction times were not affected by the training in the current study, nor was the MMN response, which was largest at the frontal area and was very similar in the pre- and post-training sessions. This result is not in line with the previous findings where the three-day training improved $d$ ' and RTs and increased MMN responses in young adults [7,8]. The ID, GR, RT, $d$ ', and MMN measures constitute a perceptual continuum in the sense that the most attention demanding perception lies in the ID and GR end. The $d$ ' and RTs, in turn, require less attentive processing approaching the pre-attentively generated MMN response. Training-related changes occurred in the self-paced ID and GR test with no time limit, which requires more effort and some earlier linguistic knowledge, compared to the oddball discrimination task measuring RTs and $d$ '. The self-paced characteristic of the task may also have been beneficial for the elderly. Even though pre-attentive changes were absent in the elderly, learning effects were evident at the behavioural level, consistent with the findings with linguistically oriented seniors [21]. The elderly participants are most probably able to benefit from 'experience-based linguistic knowledge' [26] in the more attentive perception, and hence training effects were seen in the ID and GR. As Antoniou et al. [26] summarises the suggestions of Knowles [27], the age-related experience, in general, distinguishes adult learners from child learners as they "benefit from experiential learning, they have already 
acquired the basic skills needed to succeed in life and absorb information on a 'need to know' basis, and adults are more performance-centred in their learning".

Antoniou and colleagues [26] discuss reasons why traditional learning situations may not be suitable for older learners. These include, for example, health issues such as cognitive decline, and auditory and visual problems. Furthermore, tasks which require memorization, rote, and speed may not be the best alternatives [26], as shown by the RT and $d$ ' task in the present study. With respect to that, different training methods could be considered more suitable for the elderly. First, the training rate could be slower, with more sessions or more training per session. Second, guidance in between training could also be beneficial. Third, the acoustic difference between the trained stimuli could be larger than for other age groups. More suitable training methods designed for seniors are important, not only because the older adult brain is plastic and language learning improves functions related to language, but because it also improves cognitive functions [26]. Further, since slightly declined hearing sensitivity can worsen by reduced cognitive abilities [28], it is important to improve cognitive functions.

Precision in perceiving rapid transitions of the spectrotemporal features in speech is essential for speech perception. Older people have difficulties extracting fine temporal detail in speech [17-19] and non-speech [e.g., 29] compared to younger people. It may well be that there were no training effects seen in our study because of the acoustic complexity and theoretical difficulty $[22,23]$ of the stimuli. This could explain the non-existent influence of the training on MMN in the current study, whereas our previous studies in young adults showed traininginduced MMN changes [7,8].

There are a few limitations in our study. First, the sample size could have been larger and a control group could have been helpful. However, when testing pre and post training, the participants serve as their own controls. Another limitation is that we only indirectly compared 
the elderly to younger adults. Further, the future studies should determine the influence of more extensive training and more intensive training, and comparing for example a group exposed to more training to one provided with feedback.

\section{Conclusions}

In contrast to monolingual adults and advanced L2 university students in whom training effects were previously shown both in pre-attentive neural and behavioural perception, the elderly in the current study only showed training effects in the behavioural ID and GR. In other words, changes were evident at the behavioural processing level, but not at the pre-attentive level, which implies that pre-attentive and behavioural processing do not always go hand in hand. Experience-based linguistic knowledge definitely benefits the elderly since training effects were evident in the most attention demanding tasks, while the effects were not detected in the more subconscious perception measures.

\section{Author contributions}

Henna Tamminen: Conceptualization, Methodology, Formal analysis, Investigation, Data Curation, Writing - Original Draft, Writing - Review \& Editing, Visualization, Supervision, Project administration, Funding acquisition

Teija Kujala: Data Curation, Writing - Review \& Editing

Risto Näätänen: Data Curation, Writing - Review \& Editing

Maija S. Peltola: Conceptualization, Methodology, Formal analysis, Investigation, Data Curation, Writing - Review \& Editing, Supervision, Project administration

\section{Declarations of interest}


None.

\section{Acknowledgements}

The authors thank all the subjects who participated, MA Katri Jähi for her valuable help in data collection and MA Antti Saloranta for his comments on the manuscript.

Funding: This work was supported by the Emil Aaltonen Foundation.

\section{References}

[1] L. Taimi, K. Jähi, P. Alku, M.S. Peltola, Children Learning a Non-native Vowel - The Effect of a Two-day Production Training, J. Lang. Teach. Res. 5 (2014) 1229-1235. https://doi.org/10.4304/jltr.5.6.1229-1235.

[2] W.F.L. Heeren, M.E.H. Schouten, Perceptual development of the Finnish / t-t:/ distinction in Dutch 12-year-old children: A training study, J. Phon. 38 (2010) 594-603. https://doi.org/10.1016/j.wocn.2010.08.005.

[3] A. Giannakopoulou, M. Uther, S. Ylinen, Enhanced plasticity in spoken language acquisition for child learners: Evidence from phonetic training studies in child and adult learners of English, Child Lang. Teach. Ther. 29 (2013) 201-218. https://doi.org/10.1177/0265659012467473.

[4] L. Taimi, P. Alku, T. Kujala, R. Näätänen, M.S. Peltola, The effect of production training on non-native speech sound perception and discrimination in school-aged children: An MMN and behavioural study, Linguist. Lettica. 2 (2014) 114-129.

[5] K.U. Peltola, H. Tamminen, P. Alku, M.S. Peltola, Non-native production training with an acoustic model and orthographic or transcription cues, in: Proc. 18th Int. Congr. Phonetic Sci., Glasgow, 2015. https://www.internationalphoneticassociation.org/icphsproceedings/ICPhS2015/Papers/ICPHS0236.pdf.

[6] A. Saloranta, H. Tamminen, P. Alku, M.S. Peltola, Learning of a non-native vowel through instructed production training, in: Proc. 18th Int. Congr. Phonetic Sci., Glasgow, 2015. https://www.internationalphoneticassociation.org/icphsproceedings/ICPhS2015/Papers/ICPHS0235.pdf.

[7] H. Tamminen, M.S. Peltola, T. Kujala, R. Näätänen, Phonetic training and non-native speech perception - New memory traces evolve in just three days as indexed by the mismatch negativity (MMN) and behavioural measures, Int. J. Psychophysiol. 97 (2015) 23-29. https://doi.org/10.1016/j.ijpsycho.2015.04.020.

[8] H. Tamminen, M.S. Peltola, Non-native memory traces can be further strengthened by short term phonetic training, in: Proc. 18th Int. Congr. Phonetic Sci., Glasgow, 2015. 
https://www.internationalphoneticassociation.org/icphsproceedings/ICPhS2015/Papers/ICPHS0285.pdf.

[9] R.A. Wise, Dopamine, learning and motivation, Nat. Rev. Neurosci. 5 (2004) 483-494. https://doi.org/10.1038/nrn1406.

[10] D.C. Park, P. Reuter-Lorenz, The adaptive brain: aging and neurocognitive scaffolding., $\begin{array}{lllll}\text { Annu. } & \text { Rev. } & \text { Psychol. } & 60 & \text { (2009) }\end{array}$ https://doi.org/10.1146/annurev.psych.59.103006.093656.

[11] P.C.M. Wong, J.X. Jin, G.M. Gunasekera, R. Abel, E.R. Lee, S. Dhar, Aging and cortical mechanisms of speech perception in noise, Neuropsychologia. 47 (2009) 693-703. https://doi.org/10.1016/j.neuropsychologia.2008.11.032.

[12] S. Geva, P.S. Jones, J.T. Crinion, C.J. Price, J.-C. Baron, E.A. Warburton, The effect of aging on the neural correlates of phonological word retrieval, J. Cogn. Neurosci. 24 (2012) 2135-2146. https://doi.org/10.1162/jocn_a_00278.

[13] M.A. Nippold, P.M. Cramond, C. Hayward-Mayhew, Spoken language production in adults: Examining age-related differences in syntactic complexity, Clin. Linguist. Phon. 28 (2014) 195-207. https://doi.org/10.3109/02699206.2013.841292.

[14] N.A. Dennis, R. Cabeza, Age-related dedifferentiation of learning systems: an fMRI study of implicit and explicit learning, Neurobiol. Aging. 32 (2011) 2318.e17-2318.e30. https://doi.org/10.1016/J.NEUROBIOLAGING.2010.04.004.

[15] A. Mougias, F. Christidi, M. Synetou, I. Kotrotsou, P. Valkimadi, A. Politis, Differential effect of demographics, processing speed, and depression on cognitive function in 755 non-demented community-dwelling elderly individuals, Cogn. Behav. Neurol. 32 (2019) 236-246. https://doi.org/10.1097/WNN.0000000000000211.

[16] T.H. Machado, H.C. Fichman, E.L. Santos, V.A. Carvalho, P.P. Fialho, A.M. Koenig, C.S. Fernandes, R.A. Lourenço, E.M. de P. Paradela, P. Caramelli, Normative data for healthy elderly on the phonemic verbal fluency task - FAS, Dement. Neuropsychol. 3 (2009) 55-60. https://doi.org/10.1590/S1980-57642009DN30100011.

[17] A. Strouse, D.H. Ashmead, R.N. Ohde, D.W. Grantham, Temporal processing in the aging auditory system, J. Acoust. Soc. Am. 104 (1998) 2385-2399.

[18] K.L. Tremblay, M. Piskosz, P. Souza, Aging alters the neural representation of speech cues, Neuroreport. 13 (2002) 1865-1870.

[19] T.J. Bellis, T. Nicol, N. Kraus, Aging affects hemispheric asymmetry in the neural representation of speech sounds., J. Neurosci. $20 \quad$ (2000) 791-797. https://doi.org/10632608.

[20] S.E. Kuchinsky, J.B. Ahlstrom, S.L. Cute, L.E. Humes, J.R. Dubno, M.A. Eckert, Speech-perception training for older adults with hearing loss impacts word recognition $\begin{array}{lllll}\text { and } & \text { effort, } & \text { Psychophysiology. } & 51 & \text { (2014) }\end{array}$ https://doi.org/10.1111/psyp.12242.Speech-perception.

[21] K. Jähi, M.S. Peltola, P. Alku, Does interest in language learning affect the non-native phoneme production in elderly learners?, in: Proc. 18th Int. Congr. Phonetic Sci., Glasgow, 2015. https://www.internationalphoneticassociation.org/icphs- 
proceedings/ICPhS2015/Papers/ICPHS0234.pdf.

[22] C.T. Best, W. Strange, Effects of phonological and phonetic factors on cross-language perception of approximants, J. Phon. 20 (1992) 305-330.

[23] J.E. Flege, The production of "new" and "similar" phones in a foreign language: evidence for the effect of equivalence classification, J. Phon. 15 (1987) 47-65.

[24] T. Kujala, M. Tervaniemi, E. Schröger, The mismatch negativity in cognitive and clinical neuroscience: Theoretical and methodological considerations, Biol. Psychol. 74 (2007) 1-19. https://doi.org/10.1016/j.biopsycho.2006.06.001.

[25] R.C. Oldfield, The assessment and analysis of handedness: The Edinburgh inventory, Neuropsychologia. 9 (1971) 97-113. https://doi.org/10.1016/0028-3932(71)90067-4.

[26] M. Antoniou, G.M. Gunasekera, P.C.M. Wong, Foreign language training as cognitive therapy for age-related cognitive decline: A hypothesis for future research, Neurosci. Biobehav. Rev. 37 (2013) 2689-2698. https://doi.org/10.1016/j.neubiorev.2013.09.004.

[27] M.S. Knowles, The modern practice of adult education: From pedagogy to andragogy, Follett, Chigago, 1973.

[28] S. Anderson, N. Kraus, Auditory Training: Evidence for Neural Plasticity in Older Adults, Perspect. Hear. Hear. Disord. Res. Res. Diagnostics. 17 (2013) 37-57. https://doi.org/10.1044/hhd17.1.37.

[29] J.M. Ostroff, K.L. McDonald, B.A. Schneider, C. Alain, Aging and the processing of sound duration in human auditory cortex, Hear. Res. 181 (2003) 1-7. https://doi.org/10.1016/S0378-5955(03)00113-8.

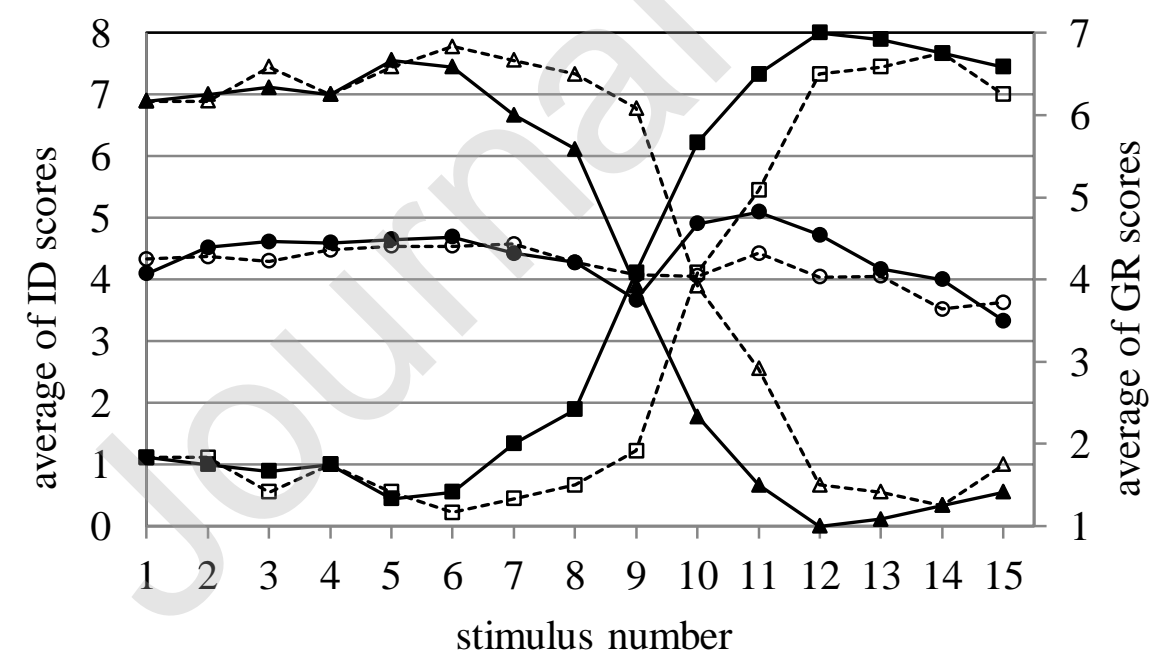

$\begin{array}{ll}\text {-------/fi:1/ - pre-training } & \longrightarrow \text { /fi:1/ - post-training } \\ \text {---日---/vi:1/ - pre-training } & \longrightarrow \text { - vi:1/ - post-training } \\ \text {------ GR - pre-training } & \longrightarrow \text { GR - post-training }\end{array}$ 
Figure 1. ID and GR scores. $X$-axis shows the 15 stimuli in the continuum, 1 was voiceless and 15 voiced; the left $Y$-axis shows how many times a stimulus was identified as a category member (max 8 times); and the right $Y$-axis is the GR scale (1=poor, $7=e x c e l l e n t)$. The standard deviations for the boundary location and steepness are presented in Table 2.

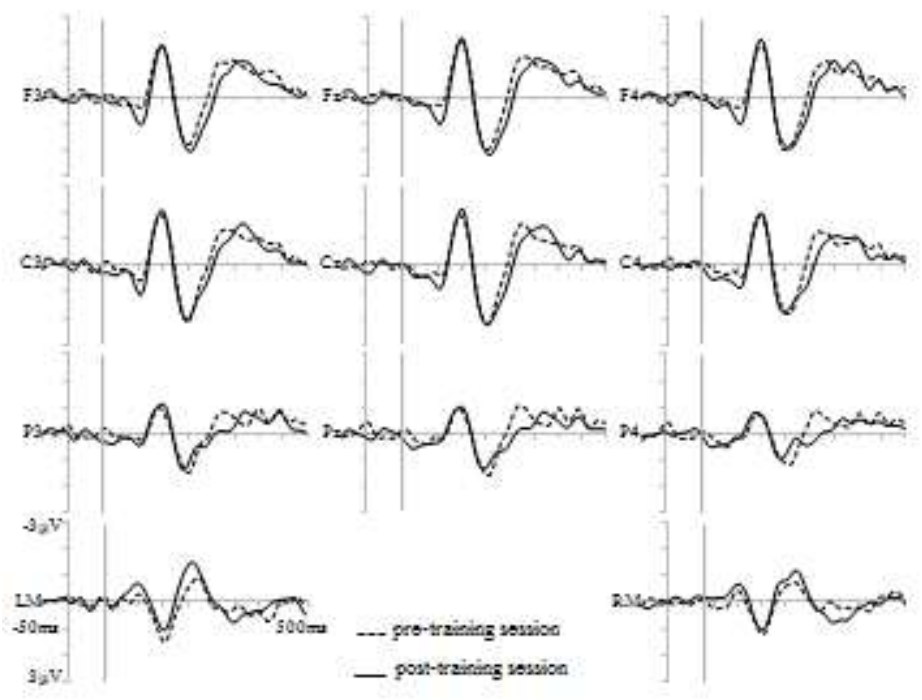

Figure 2. The grand average difference waveforms from pre- and post-training sessions. The difference between the stimuli starts at $71 \mathrm{~ms}$ (the second vertical line from left).

Table 1. The years English has been studied during primary education, how much English has been heard daily, and how well English has been mastered in speaking, understanding, reading and writing situations.

\begin{tabular}{llllll} 
& O years & 3 years & 4 years & 5 years & 7 years \\
\cline { 2 - 5 } English studied & 3 & 2 & 1 & 1 & 3 \\
\hline \multirow{2}{*}{$\begin{array}{l}\text { English } \\
\text { daily }\end{array}$} & not at all & little & quite a lot & a lot & \\
\hline & 0 & 6 & 4 & 0 & excellent \\
\hline
\end{tabular}




\begin{tabular}{llllll}
\cline { 2 - 4 } & 3 & 1 & 5 & 1 & 0 \\
$\begin{array}{l}\text { master } \\
\text { understanding }\end{array}$ & 3 & 0 & 5 & 2 & 0 \\
master reading & 3 & 0 & 6 & 1 & 0 \\
master writing & 3 & 1 & 6 & 0 & 0
\end{tabular}

Table 2. The mean category boundary location and steepness in the ID; the mean GR values for stimuli 7-10; the mean $d$ ' and RT values; and the mean MMN amplitudes for time window 300-360 ms. Standard deviations are in brackets.

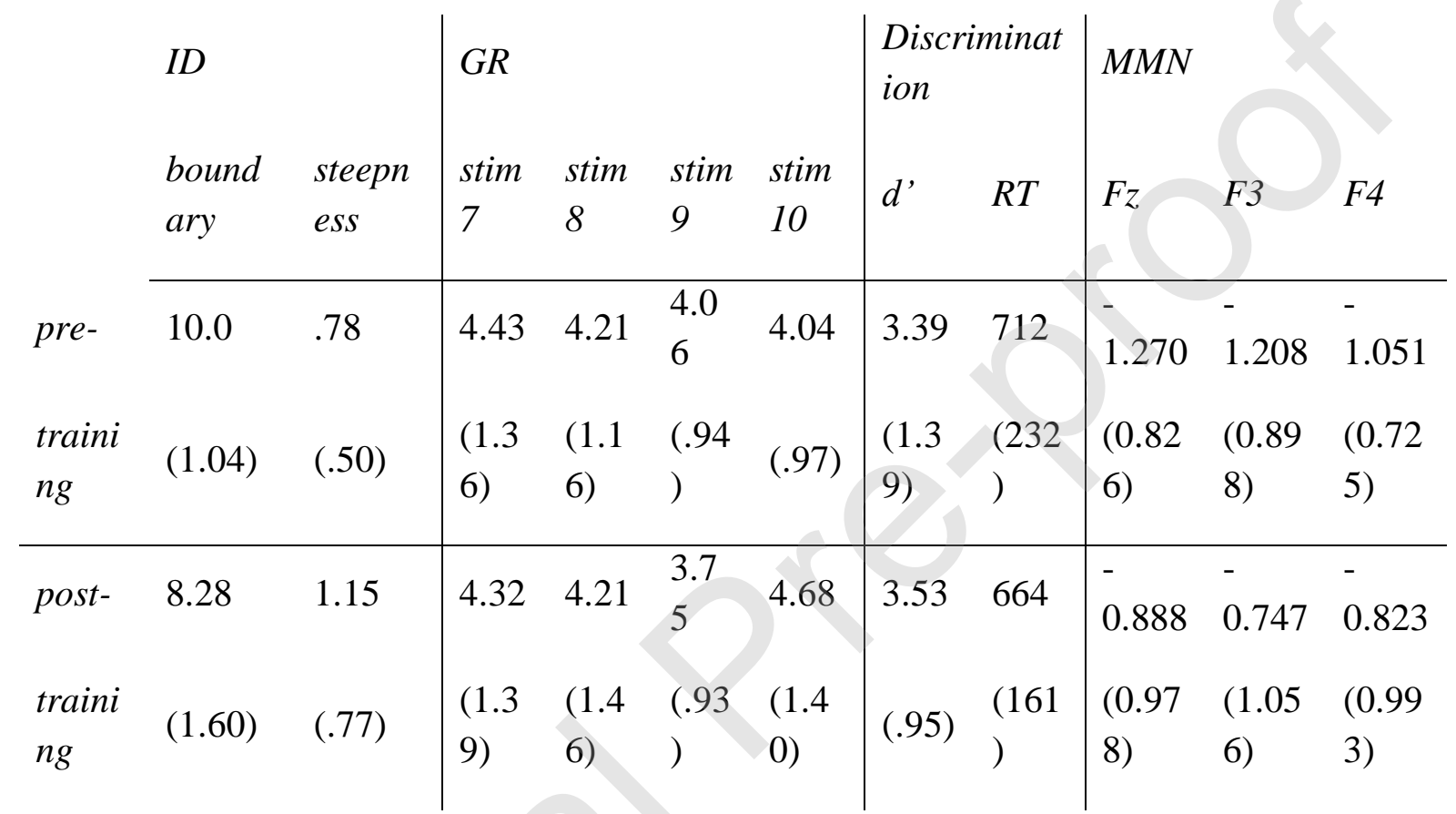

